\title{
VIP-Secreting Ganglioneuroblastoma as an Unusual Cause of Watery Diarrhoea in Childhood
}

\author{
Koen Huysentruyt ${ }^{\mathrm{a}}$, Wim Arts ${ }^{\mathrm{b}, \mathrm{c}}$, Jutte van der Werff ten Bosch ${ }^{\mathrm{a}}$, Willy Van de Casseye ${ }^{\mathrm{b}}$, \\ Francis Lemmens $^{\mathrm{b}}$, Jan De Koster ${ }^{\mathrm{b}}$, Yvan Vandenplas ${ }^{\mathrm{a}}$, An Van Damme ${ }^{\mathrm{a}}$
}

\begin{abstract}
A 15-month-old boy with a 2-month history of watery diarrhoea presented with cachexia, hypokalaemia, hypochloraemia and metabolic acidosis. During a 2 -week investigation period infection, celiac disease and cystic fibrosis were excluded and trial therapy with antibiotics, probiotics and dietary measures proved unsuccessful. This led to the suspected diagnosis of a vasoactive intestinal polypeptide (VIP)-secreting tumour with watery diarrhoea, hypokalaemia, achlorhydria (WDHA)-syndrome. MRI showed an infrarenal mass infiltrating the neuroforamen, which on pathological examination was consistent with a VIP-secreting neuroganglioblastoma. Serum VIP and urinary catecholamine levels were elevated. Treatment consisted of 2 courses of chemotherapy and a partial resection of the tumour, following which the diarrhoea disappeared. Refractory watery diarrhoea that persists during fasting in a young child should alert to the diagnosis of WDHA, and prompt investigations to diagnose a VIP-secreting tumour, one of the most common causes of secretory diarrhoea in the developed world. Timely diagnosis could avoid morbidity due to unnecessary investigations and protracted diarrhoea.
\end{abstract}

Keywords: Child; Ganglioneuroblastoma; Neuroblastoma; Secretory diarrhoea; Vasoactive intestinal polypeptide; Vipoma; WDHA syndrome

\section{Introduction}

A syndrome of profuse watery diarrhoea, hypokalaemia and

Manuscript accepted for publication January 2, 2013

\footnotetext{
${ }^{\text {a } D e p a r t m e n t ~ o f ~ P e d i a t r i c s, ~ U n i v e r s i t y ~ H o s p i t a l, ~ B r u s s e l s ~ F r e e ~}$ University, Brussels, Belgium

${ }^{\mathrm{b}}$ Department of Pediatrics, Ziekenhuis Oost-limburg, Genk, Belgium

${ }^{\mathrm{c}}$ Corresponding author: Wim Arts, Department of Pediatrics,

Ziekenhuis Oost-limburg, Schiepse Bos 6, 3600 Genk, Belgium.

Email:wim.arts@zol.be
}

doi: http://dx.doi.org/10.4021/jmc1009w achlorhydria was first described by Verner and Morrison in 1958 in 2 adults with a non-beta islet cell adenoma [1]. Bloom et al where the first to link this condition with high serum vasoactive intestinal polypeptide (VIP) levels in 5 patients with a pancreatic tumour, but also in a case was with a ganglioneuroblastoma [2]. The syndrome was later also called 'vipoma syndrome', or 'watery diarrhoea, hypochloremia and acidosis syndrome' (WDHA), since it is now generally agreed that achlorhydria is not an essential part of the syndrome and that hypochlorhydria or even normal chlorhydric levels may exist $[3,4]$. In children WDHA is very rare, and although excessive VIP secretion is well described in neuroblastic tumours, it occurs in less than $1 \%$ of cases $[5,6]$. We will discuss that the rareness of vipoma-induced WDHA in childhood renders diagnosis difficult, leading to unnecessary investigations, a delay in diagnosis and increased morbidity in these children. We will also point out some important clues that could lead to a more timely diagnosis.

\section{Case Report}

A 15-month-old boy presented with a two-month history of watery diarrhoea with no other associated symptoms. Treatment had consisted of oral rehydration solution during exacerbations while probiotic and antibiotic therapy remained without effect. The boy was born at 36 weeks gestation (birth weight $2.650 \mathrm{~kg}$ ). There was no family history of any intestinal or endocrine disorders. He was fed on formula milk since the age of 2 weeks; vegetables were introduced at the age of 3.5 months, fruits at 4.5 and gluten at 9 months. He had received all standard childhood immunizations.

When the child's weight deviated from the 25th to the 3rd centile, he was hospitalized for further investigations. On admission, clinical exam showed a pale and cachectic toddler, with a soft and non-tender abdomen without organomegaly, distension or palpable masses. Further systemic examination revealed no anomalies. Blood pressure was $114 / 57 \mathrm{mmHg}$ (>99th centile). Lab analysis showed hypokalaemia $(2.4 \mathrm{mmol} / \mathrm{L})$, hypochlorhydria $(96 \mathrm{mmol} / \mathrm{L})$ and metabolic acidosis (bicarbonate $10 \mathrm{mmol} / \mathrm{L}$ ); sodium levels were normal. Haemoglobin was $12.2 \mathrm{~g} / \mathrm{dL}$; total white cell 


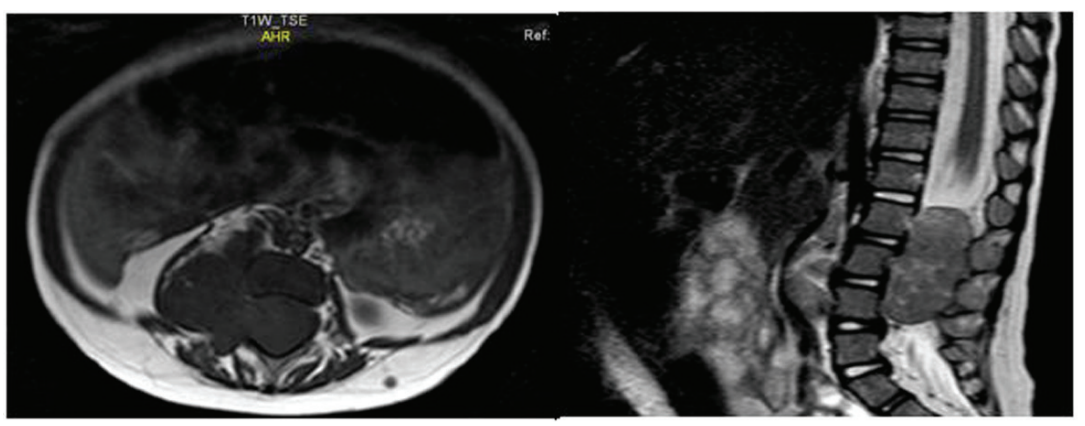

Figure 1. MRI of the lumbal region showing a paravertebral mass infiltrating the spinal channel.

count $18.6 \times 10^{9} / \mathrm{L}$ and platelet count $937 \times 10^{9} / \mathrm{L}$; ESR was $56 \mathrm{~mm} / \mathrm{h}$. The total serum IgA level was normal. Repeated stool examinations were negative for pathogenic bacteria or viruses. Abdominal ultrasound was normal. Gastroscopy showed no abnormalities. Apart from subtle intra-epithelial lymphocytosis, a small-bowel biopsy was unremarkable and with anti-tissue transglutaminase antibodies being negative, celiac disease was ruled out. A left colonoscopy showed macro- and microscopically normal findings. Cystic fibrosis genotyping (InnoLipa CFTR19, CFTR17+Tn Update) showed no mutation. During the course of the investigations, dietary elimination of cow's milk protein and lactose was ineffective. With common causes of chronic diarrhoea being ruled out, the persistence of watery diarrhoea raised the suspicion of a VIP-secreting tumour. Urine Dopamine and HVA were elevated $(8,643 \mu \mathrm{g} / \mathrm{g} \mathrm{Cr}$ and $39.7 \mathrm{mg} / \mathrm{g} \mathrm{Cr}$ respectively), whilst urine VMA was normal $(14.4 \mathrm{mg} / \mathrm{g}$ Cr). Serum-VIP level was significantly elevated (187 pg/ $\mathrm{mL}$ ). MRI showed a right-sided infrarenal mass infiltrating the neuroforamen (L3-L4) with a mass effect on the spinal cord (Fig. 1), and pathology of a biopsy confirmed the diagnosis of a VIP-secreting ganglioneuroblastoma. In view of massive intraspinal infiltration, the boy received 2 courses of chemotherapy (Carboplatinum, VP16), failing to result in shrinkage of tumour. Subtotal resection of the tumour was performed. Diarrhoea transiently improved after each chemotherapy course, and fully disappeared after surgery. One year after surgery, catch-up growth and weight gain has occurred to the 25 th and 3 rd centile respectively.

\section{Discussion}

This case is illustrated how the rarity of the WDHA syndrome may lead to an initial denial of the diagnosis, even by experienced paediatric gastro-enterologists, and that a normal abdominal ultrasound may offer a false reassurance.

Chronic diarrhoea in childhood is not uncommon, and may result from a wide range of gastrointestinal disorders. The etiologies of chronic diarrhoea in childhood can be divided into four often overlapping pathophysiology mechanisms: osmotic, secretory, dysmotility-associated and inflammatory diarrhoea. True secretory diarrhoea concerns only a small proportion these cases and is the only type where diarrhoea persists even during prolonged fasting. A low stool osmotic gap (290- calculated stool osmolality = stool osmotic gap) is also a major hallmark of secretory diarrhoea, whereas a high stool osmolality and cessation of diarrhoea with elimination of the offending solute from the diet are specific for osmotic diarrhoea. Bloody stools and pathogens are seen in inflammatory diarrhoea. Dysmotility-associated diarrhoea (for example, toddler's diarrhoea) is harder to prove and is often a diagnosis of exclusion.

The large time gap between onset of diarrhoea and diagnosis of the tumour is supported by findings from 2 retrospective studies on vipoma cases in childhood. In a review of 6 patients, Murphy et al reported a median hospital stay for investigations of 4 weeks, while the interval between referral and eventual diagnosis ranged from 1 to 10 months; diarrhoea persisted for a median duration of 5 months, and 5 children were severely malnourished at the time of diagnosis [7]. These findings are consistent with those of Bourdeaut et al in a series of 22 children with VIP-secreting neuroblastic tumours [5]. Likewise, our patient's hospital stay was 2 weeks, and the definitive diagnosis was obtained 3 months after start of the diarrhoea.

We propose set of clinical features that may facilitate early diagnosis of vipoma in childhood. First, although WDHA has been described at various ages, it appears to be more common in infants and young children. The median age of presentation was 21 months in the Iida study, 18 months in the Bourdeaut study, and all cases described by Murphy et al were 24 months or younger $[5,7,8]$; our patient also falls in this age category. Second, chronic watery diarrhoea refractory to fasting and total parenteral nutrition during 48 to 72 hours should alert to the diagnosis of secretory diarrhoea and a VIP-secreting tumour. Boureaut et al reported that diarrhoea was the presenting symptom in 16 out of 22 patients. In the remaining 6, diarrhoea developed after chemotherapy, a phenomenon referred to as 'secondary VIP-secreting neuroblastic tumour', and thought to be due to the release of VIP during chemotherapy-induced tumour 
lysis. Alternatively, a more generally accepted theory holds that chemotherapy concomitantly induces differentiation of (poorly differentiated) non-VIP-secreting neuroblastic tumours, as well as their capacity to secrete VIP $[5,8]$. Third, a blood pressure over the 99th centile on repeated measurements should, especially in combination with the other signs and symptoms, should remind the clinician to the possibility of a neural crest tumour. The patient in our report also presented with hypertension, but the child reported by Swift et al. presented with a normal blood pressure [9]. Hypertension is however well known to be associated with neurogenic tumours in children [10]. Fourth, according to Iida et al, flushing may also direct to the diagnosis. In their series, flushing was noted in 7 of 31 cases. However, this is a transient sign and can easily be overlooked [8]. Last, sweating and waddling gait may also be present [6]. Our patient showed none of these signs.

In conclusion, intractable watery diarrhoea that persists during fasting in a young child should alert to the diagnosis of secretory diarrhoea. In this case a VIP secreting tumour, one of the most frequent causes of secretory diarrhoea in the developed world, should be ruled out, especially when concurrent hypertension is present. Timely diagnosis using plasma VIP levels, urinary catecholamine levels and MRI imaging should avoid unnecessary morbidity due to needless investigations and protracted diarrhoea.

\section{Acknowledgement}

We thankfully acknowledge Prof. Dr. An Billiau for the critical review of this paper.

\section{Grants and Conflict of Interest}

The authors have no conflict of interest or financial support to declare.

\section{References}

1. Verner JV, Morrison AB. Islet cell tumor and a syndrome of refractory watery diarrhea and hypokalemia. Am J Med. 1958;25(3):374-380.

2. Bloom SR, Polak JM, Pearse AG. Vasoactive intestinal peptide and watery-diarrhoea syndrome. Lancet. 1973;2(7819):14-16.

3. Grier JF. WDHA (watery diarrhea, hypokalemia, achlorhydria) syndrome: clinical features, diagnosis, and treatment. South Med J. 1995;88(1):22-24.

4. Modlin IM, Bloom SR, Mitchell SJ. Experimental evidence for vasoactive intestinal peptide as the cause of the watery diarrhea syndrome. Gastroenterology. 1978;75(6):1051-1054.

5. Bourdeaut F, de Carli E, Timsit S, Coze C, Chastagner P, Sarnacki S, Delattre O, et al. VIP hypersecretion as primary or secondary syndrome in neuroblastoma: A retrospective study by the Societe Francaise des Cancers de l'Enfant (SFCE). Pediatr Blood Cancer. 2009;52(5):585590.

6. Leleiko NS, Gee MS, Sadow PM. Case records of the Massachusetts General Hospital. Case 13-2010. An 18.5-month-old girl with watery diarrhea and poor weight gain. N Engl J Med. 2010;362(17):1619-1626.

7. Murphy MS, Sibal A, Mann JR. Persistent diarrhoea and occult vipomas in children. BMJ. 2000;320(7248):15241526.

8. Iida Y, Nose O, Kai H, Okada A, Mori T, Lee PK, Kakudo K, et al. Watery diarrhoea with a vasoactive intestinal peptide-producing ganglioneuroblastoma. Arch Dis Child. 1980;55(12):929-936.

9. Swift PG, Bloom SR, Harris F. Watery diarrhoea and ganglioneuroma with secretion of vasoactive intestinal peptide. Arch Dis Child. 1975;50(11):896-899.

10. Weinblatt ME, Heisel MA, Siegel SE. Hypertension in children with neurogenic tumors. Pediatrics. 1983;71(6):947-951. 